PoS $\quad \begin{aligned} & \text { PROCEEDINGS } \\ & \text { OF SCIENCE }\end{aligned}$

\title{
Recent Developments in the Theory of Quarkonium
}

\section{Nora Brambilla*}

Physik-Department, Technische Universität München, James-Franck-Str. 1, 85748 Garching, Germany

E-mail: nora.brambilla@ph.tum.de

I review some recent developments in the theory of heavy quarkonium in an effective field theory framework.

The Xth Nicola Cabibbo International Conference on Heavy Quarks and Leptons, October 11-15, 2010

Frascati (Rome) Italy

${ }^{*}$ Speaker. 


\section{Quarkonium}

In the last few years the B-factories Babar at SLAC and Belle at KEK, CLEO-III and Cleo$\mathrm{c}$ at CESR, CDF and D0 at Fermilab and BESII and BESIII at IHEP have largely operated as quarkonium factories producing large data sample on quarkonium spectra, decays and production with high statistics. New states, new production mechanisms, new transitions and unexpected states of an exotic nature have been observed. The study of quarkonium in media has also undergone crucial developments, the suppression of quarkonium production in the hot medium remaining one of the cleanest and most relevant probe of deconfined matter. More is expected to come soon from LHC experiments.

From the theory point of view, effective field theories (EFTs) as HQEFT, NRQCD, pNRQCD, SCET..., for the description of quarkonium processes have been newly developed and are being developed, providing a unifying description as well as a solid and versatile tool giving well-defined, model independent and precise predictions $[1,2]$. They rely on one hand on high order perturbative calculations and on the other hand on lattice simulations, the recent progress in both fields having added a lot to the theory reach.

The International Quarkonium Working Group (QWG) (www.qwg.to.infn.it) created in 2002 has supplied ad adequate platform for discussion and common researrch work between theorists and experimentalists, producing also two large reviews of state of the art, open problems, perspective and opportunities of quarkonium physics in 2010 and 2004 [1].

Here I will summarize some of the recent progress in theory.

\section{Effective Field Theories for Quarkonium}

The modern approach to quarkonium physics consists in taking advantage of the hierarchy of non-relativistic (NR) energy scales in the system by constructing a suitable hierarchy of effective field theories (EFTs) [2].

The energy scales are: the heavy-quark mass (hard scale), $m$, the typical momentum transfer (soft scale), $p \sim m v$, whose inverse sets the typical distance, $r$, between the heavy quark and the antiquark, and the typical kinetic energy (ultrasoft scale), $E \sim m v^{2}$, whose inverse sets the typical time scale of the bound state. The heavy-quark bound-state velocity $v$ is a small quantity $v \ll 1\left(v^{2} \sim 0.1\right.$ for $b \bar{b}, v^{2} \sim 0.3$ for $c \bar{c}, v^{2} \sim 0.01$ for $\left.t \bar{t}\right)$, the mass is a large quantity $m \gg \Lambda_{\mathrm{QCD}}, \alpha_{\mathrm{S}}(m) \ll 1$. For energy scales close to $\Lambda_{\mathrm{QCD}}$, perturbation theory breaks down and one has to rely on nonperturbative methods. Regardless of this, the nonrelativistic hierarchy of scales: $m \gg p \sim 1 / r \sim m v \gg E \sim m v^{2}$ also persists below the $\Lambda_{\mathrm{QCD}}$ threshold. While the hard scale is always larger than $\Lambda_{\mathrm{QCD}}$, different situations may arise for the other two scales depending on the considered quarkonium system. The soft scale, proportional to the inverse typical radius $r$, may be a perturbative ( $\gg \Lambda_{\mathrm{QCD}}$ ) or a nonperturbative scale $\left(\sim \Lambda_{\mathrm{QCD}}\right)$ depending on the physical system. The first case is likely to happen only for the lowest charmonium and bottomonium states. We do not have direct information on the radius of the quarkonia systems, and thus the attribution of some of the lowest bottomonia and charmonia states to the perturbative or the nonperturbative soft regime is at the moment still ambiguous. The ultrasoft scale may still be perturbative only in the case of $t \bar{t}$ threshold states. All quarkonium scales get entangled in a typical amplitude involving a quarkonium observable. In 
particular, quarkonium annihilation and production take place at the scale $m$, quarkonium binding takes place at the scale $m v$, which is the typical momentum exchanged inside the bound state, while very low-energy gluons and light quarks (also called ultrasoft degrees of freedom) live long enough that a bound state has time to form and, therefore, are sensitive to the scale $m v^{2}$. Ultrasoft gluons are responsible for phenomena like the Lamb shift in QCD.

A hierarchy of EFTs may be constructed by systematically integrating out modes associated to high energy scales not relevant for quarkonium. Such integration is made in a matching procedure enforcing the equivalence between QCD and the EFT at a given order of the expansion in $v$. The EFT realizes a factorization at the Lagrangian level between the high energy contributions, encoded into the matching coefficients, and the low energy contributions, carried by the dynamical degrees of freedom. Poincare symmetry remains intact in a nonlinear realization at the level of the NR EFT and imposes exact relations among the matching coefficients [3].

\section{Physics at the scale $m$ : NRQCD}

At the scale $m$ the suitable EFT is NonRelativistic QCD (NRQCD) [4], which follows from QCD by integrating out the scale $m$. As a consequence, the effective Lagrangian is organized as an expansion in $1 / m$ and $\alpha_{\mathrm{s}}(m)$. The field of quarkonium production has seen terrific progress in the last few years both in theory and in experiments, for a review see [1, 5].

For what concerns decays, recently, substantial progress has been made in the evaluation of the factorization formula at order $v^{7}[6]$, in the lattice evaluation of the NRQCD matrix elements [7] and in the new, accurate data on many hadronic and electromagnetic decays [1]. The data are clearly sensitive to NLO corrections in the Wilson coefficients and presumably also to relativistic corrections. Improved theory predictability would entail the lattice calculation or data extraction of the NRQCD matrix elements and perturbative resummation of large contribution in the NRQCD matching coefficients. The new data on hadronic transitions and hadronic decays pose interesting challenging to the theory.

Using the new CLEO data on radiative $\Upsilon(1 S)$ decay and the improved lattice determination of the NRQCD matrix elements it has been possible to obtain in [8] a determination of $\alpha_{\mathrm{s}}$ at the $\Upsilon$ mass $\alpha_{\mathrm{s}}\left(M_{\Upsilon}(1 S)\right)=0.184_{-0.014}^{+0.015}$ giving a value $\alpha_{\mathrm{s}}\left(M_{z}\right)=0.119_{-0.005}^{0.006}$ in agreement with the world average.

Lattice NRQCD calculations have undergone a steady development in last few years see [1,9].

\section{Physics at the scale $m v$ and $m v^{2}: \mathbf{p N R Q C D}$}

At the scales $m v$ and $m v^{2}$ the suitable EFT is potential NonRelativistic QCD (pNRQCD) [10, 11], which follows from NRQCD by integrating out the scale $m v$.

For quarkonium states away from threshold we have now a clear effective field description, based on perturbative and lattice computations. This is nowadays the standard description.

The soft scale $m v$ may be larger or not than the confinement scale $\Lambda_{\mathrm{QCD}}$ depending on the radius of the quarkonium system. When $m v^{2} \sim \Lambda_{\mathrm{QCD}}$, we speak about weakly-coupled pNRQCD because the soft scale is perturbative and the matching from NRQCD to pNRQCD may be performed in perturbation theory. When $m v \sim \Lambda_{\mathrm{QCD}}$, we speak about strongly-coupled pNRQCD 
because the soft scale is nonperturbative and the matching from NRQCD to PNRQCD may not be performed in perturbation theory [2].

The potential is a Wilson coefficient of the EFT. In general undergoes renormalization, develops scale dependence and satisfies renormalization group equations which allow to resum large logarithms. In this framework the $Q \bar{Q}$ potential, which is a fundamental object for QCD, can be defined and systematically calculated.

In the following we will summarize some recent phenomenological applications of pNRQCD. There are several cases for the physics at hand. In the case in which the EFT has been constructed $[11,12,2]$, i.e. for states below threshold, the work is currently going in calculating higher order perturbative corrections in $v$ and $\alpha_{\mathrm{s}}$ for processes of interest, resumming the logarithms in the ratio of the scales that may be sizeable, calculating or extracting nonperturbatively low energy correlators and extending the theory with the addictions of electromagnetic effects [13] and the consideration of $Q Q Q$ and $Q Q q$ systems [14]. The issue here is precision physics and the study of confinement. Close to threshold the full EFT has not yet been constructed and the degrees of freedom have still to be identified $[15,1]$. At finite temperature the EFT is being constructed and the existing results hint at a new physical picture with possible application at heavy ion collisions at LHC.

\subsubsection{Potentials, spectrum, decays for quarkonia of small radius}

If the quarkonium system is small, the soft scale is perturbative and the potentials can be entirely calculated in perturbation theory [2].

Since the degrees of freedom that enter the Schrödinger description are in this case both $Q \bar{Q}$ color singlet and $Q \bar{Q}$ color octets, both singlet and octet potentials exist. The static singlet $Q \bar{Q}$ potential is pretty well known. The three-loop correction to the static potential is now completely known: the fermionic contributions to the three-loop coefficient [16] first became available, and more recently the remaining purely gluonic term has been obtained $[17,18]$.

The first log related to ultrasoft effects arises at three loops [19] . Such logarithm contribution at $\mathrm{N}^{3} \mathrm{LO}$ and the single logarithm contribution at $\mathrm{N}^{4} \mathrm{LO}$ may be extracted respectively from a oneloop and two-loop calculation in the EFT and have been calculated in [20, 21].

The perturbative series of the static potential suffers from a renormalon ambiguity (i.e. large $\beta_{0}$ contributions) and from large logarithmic contributions. The singlet static energy, given by the sum of a constant, the static potential and the ultrasoft corrections, is free from ambiguities of the perturbative series. By resumming the large logs using the renormalization group equations and comparing it (at the NNLL) with lattice calculations of the static energy one sees that the QCD perturbative series converges very nicely to and agrees with the lattice result in the short range (up to $0.25 \mathrm{fm}$ ) and that no nonperturbative linear ("stringy") contribution to the static potential exist $[22,20]$.

In particular, the recently obtained theoretical expression [20] for the complete QCD static energy at NNNLL precision has been used to determine $r_{0} \Lambda_{\overline{M S}}$ by comparison with available lattice data, where $r_{0}$ is the lattice scale and $\Lambda_{\bar{M} S}$ is the QCD scale, obtaining $r_{0} \Lambda_{\bar{M} S}=0.622_{-0.015}^{+0.019}$ for the zero-flavor case. This extraction was previously performed at the NNLO level (including an estimate at NNNLO) in [23]. The same procedure can be used to obtain a precise evaluation of the unquenched $r_{0} \Lambda_{\bar{M} S}$ value after short distance unquenched lattice data for the $Q \bar{Q}$ exist [24]. 
The static octet potential is known up to two loops [25]. Relativistic corrections to the static singlet potential have been calculated over the years and are summarized in [2].

In the case of $Q Q q$ baryons, the static potential has been determined up to NNLO in perturbation theory [14] and recently also on the lattice [26]. Terms suppressed by powers of $1 / m$ and $r$ in the Lagrangian have been matched (mostly) at leading order and used to determine, for instance, the expected hyperfine splitting of the ground state of these systems.

In the case of $Q Q Q$ baryons, the static potential has been determined up to NNLO in perturbation theory [14] and also on the lattice [27]. The transition region from a Coulomb to a linearly raising potential is characterized in this case also by the emergence of a three-body potential apparently parameterized by only one length. It has been shown that in perturbation theory a smooth genuine three-body potential shows up at two loops.

The energy levels have been calculated at order $m \alpha_{\mathrm{s}}^{5}$ [29]. Decays amplitude [28, 1, 2] and production and annihilation [30] have been calculated in perturbation theory at high order. An effective field theory of magnetic dipole transition has been given in [13] and a description of the $\eta_{c}$ line shape in [31].

\subsubsection{Potentials, spectra and decays for quarkonia of large radius}

If the quarkonium system is large, the soft scale is nonperturbative and the potentials cannot be entirely calculated in perturbation theory [2]. Then the potential matching coefficients are obtained in the form of expectation values of gauge-invariant Wilson-loop operators. In this case, heavylight meson pairs and heavy hybrids develop a mass gap of order $\Lambda_{\mathrm{QCD}}$ with respect to the energy of the $Q \bar{Q}$ pair, the second circumstance being apparent from lattice simulations. Thus, away from threshold, the quarkonium singlet field $S$ is the only low-energy dynamical degree of freedom in the pNRQCD Lagrangian (neglecting ultrasoft corrections coming from pions and other Goldstone bosons). The singlet potential $V_{S}(r)$ can be expanded in powers of the inverse of the quark mass; static, $1 / \mathrm{m}$ and $1 / \mathrm{m}^{2}$ terms were calculated long ago [12]. They involve NRQCD matching coefficients (containing the contribution from the hard scale) and low-energy nonperturbative parts given in terms of static Wilson loops and field-strength insertions in the static Wilson loop (containing the contribution from the soft scale). Such expressions correct and generalize previous finding in the Wilson loop approach [32] that were typically missing the high energy parts of the potentials, encoded into the NRQCD matching coefficients and containing the dependence on the logarithms of $m_{Q}$, and some of the low energy contributions.

In this regime of pNRQCD, we recover the quark potential singlet model. However, here the potentials are calculated in QCD by nonperturbative matching. Their evaluation requires calculations on the lattice or in QCD vacuum models [33]. Recent progress includes new, precise lattice calculations of these potentials obtained using the Lüscher multi-level algorithm [34]. The nonperturbative potentials for the $Q Q Q$ and $Q Q q$ have been obtained in the second reference of [14] and in [35]. Inclusive decay amplitudes have been calculated (see the last ref. in [12]) and the number of nonperturbative correlators appears to be sizeably reduced with respect to NRQCD.

\subsection{Quarkonium potential at finite $T$ and heavy ion collisions}

In the last few years years, there has been a remarkable progress in constructing EFTs for quarkonium at finite temperature and in rigorously defining the quarkonium potential $[36,37]$. 
Quarkonium in a medium is characterized by different energy and momentum scales; there are the scales of the non-relativistic bound state and there are the thermodynamical scales: the temperature $T$, the inverse of the screening length of the chromoelectric interactions, i.e. the Debye mass $m_{D}$ and lower scales. Up to now calculations are done in the weak coupling regime. Integrating out sequentially the energy scales a version of $\mathrm{PNRQCD}$ at finite $\mathrm{T}$ has been obtained and the potential has been calculated as matching coefficient of the EFT. Such potential has new and unexpected feautures, e.g. a large imaginary contribution, that will have an important impact in phenomenological studies of quarkonium suppression at RHIC and at LHC. In particular in [38] heavy quarkonium energy levels and decay widths in a quark-gluon plasma, below the melting temperature at a temperature $\mathrm{T}$ and screening mass $m_{D}$ satisfying the hierarchy $m \alpha_{\mathrm{s}} \gg \pi T \gg m \alpha_{\mathrm{s}}^{2} \gg m_{D}$, have been calculated at order $m \alpha_{\mathrm{s}}^{5}$. This situation is relevant for bottomonium $1 S$ states $\left(\Upsilon(1 S), \eta_{b}\right)$ at the LHC.

\section{References}

[1] N. Brambilla et al., arXiv:1010.5827 [hep-ph]; N. Brambilla et al. [Quarkonium Working Group], arXiv:hep-ph/0412158.

[2] N. Brambilla, A. Pineda, J. Soto and A. Vairo, Rev. Mod. Phys. 77, 1423 (2005)

[3] N. Brambilla, D. Gromes and A. Vairo, Phys. Lett. B 576 (2003) 314; N. Brambilla, D. Gromes and A. Vairo, Phys. Rev. D 64 (2001) 076010

[4] W. E. Caswell and G. P. Lepage, Phys. Lett. B 167 (1986) 437; G. T. Bodwin, E. Braaten and G. P. Lepage, Phys. Rev. D 51 (1995) 1125 [Erratum-ibid. D 55 (1997) 5853].

[5] G. T. Bodwin, arXiv:1012.4215 [hep-ph]; J. P. Lansberg et al., AIP Conf. Proc. 1038 (2008) 15

[6] N. Brambilla, E. Mereghetti and A. Vairo, JHEP 0608 (2006) 039; N. Brambilla, E. Mereghetti and A. Vairo, Phys. Rev. D 79 (2009) 074002

[7] G. T. Bodwin, J. Lee and D. K. Sinclair, Phys. Rev. D 72 (2005) 014009

[8] N. Brambilla, X. Garcia i Tormo, J. Soto and A. Vairo, Phys. Rev. D 75 (2007) 074014

[9] C. T. H. Davies and f. t. H. collaboration, arXiv:0810.3309 [hep-lat].

[10] A. Pineda and J. Soto, Nucl. Phys. Proc. Suppl. 64 (1998) 428.

[11] N. Brambilla, A. Pineda, J. Soto and A. Vairo, Nucl. Phys. B 566 (2000) 275.

[12] N. Brambilla, A. Pineda, J. Soto and A. Vairo, Phys. Rev. D 63 (2001) 014023; A. Pineda and A. Vairo, Phys. Rev. D 63 (2001) 054007 [Erratum-ibid. D 64 (2001) 039902]; N. Brambilla, D. Eiras, A. Pineda, J. Soto and A. Vairo, Phys. Rev. D 67 (2003) 034018

[13] N. Brambilla, Y. Jia and A. Vairo, Phys. Rev. D 73 (2006) 054005 [arXiv:hep-ph/0512369].

[14] N. Brambilla, J. Ghiglieri and A. Vairo, Phys. Rev. D 81, 054031 (2010); N. Brambilla, A. Vairo and T. Rosch, Phys. Rev. D 72 (2005) 034021.

[15] N. Brambilla, A. Vairo, A. Polosa and J. Soto, Nucl. Phys. Proc. Suppl. 185 (2008) 107.

[16] A. V. Smirnov, V. A. Smirnov and M. Steinhauser, Phys. Lett. B 668 (2008) 293,

[17] C. Anzai, Y. Kiyo and Y. Sumino, Phys. Rev. Lett. 104 (2010) 112003, 
[18] A. V. Smirnov, V. A. Smirnov and M. Steinhauser, Phys. Rev. Lett. 104 (2010) 112002 ,

[19] N. Brambilla et al., Phys. Rev. D 60 (1999) 091502.

[20] N. Brambilla, X. Garcia i Tormo, J. Soto and A. Vairo, Phys. Rev. Lett. 105 (2010) 212001

[21] N. Brambilla, A. Vairo, X. Garcia i Tormo and J. Soto, Phys. Rev. D 80 (2009) 034016; N. Brambilla, X. Garcia i Tormo, J. Soto and A. Vairo, Phys. Lett. B 647 (2007) 185.

[22] A. Pineda, J. Phys. G 29 (2003) 371.

[23] Y. Sumino, Phys. Rev. D 76 (2007) 114009,

[24] M. Donnellan, F. Knechtli, B. Leder and R. Sommer, arXiv:1012.3037 [hep-lat].

[25] B. A. Kniehl, A. A. Penin, Y. Schroder, V. A. Smirnov and M. Steinhauser, Phys. Lett. B 607 (2005) 96 ,

[26] A. Yamamoto, H. Suganuma and H. Iida, Phys. Lett. B 664 (2008) 129; Phys. Rev. D 78 (2008) 014513; J. Najjar and G. Bali, arXiv:0910.2824 [hep-lat].

[27] T. T. Takahashi, H. Matsufuru, Y. Nemoto and H. Suganuma, Phys. Rev. Lett. 86 (2001) 18; Phys. Rev. D 65 (2002) 114509.

[28] Y. Kiyo, A. Pineda and A. Signer, arXiv:1006.2685 [hep-ph].

[29] N. Brambilla, A. Pineda, J. Soto and A. Vairo, Phys. Lett. B 470, 215 (1999); B. A. Kniehl, A. A. Penin, V. A. Smirnov and M. Steinhauser, Nucl. Phys. B 635 (2002) 357

[30] M. Beneke, Y. Kiyo and A. A. Penin, Phys. Lett. B 653 (2007) 53

[31] N. Brambilla, P. Roig and A. Vairo, arXiv:1012.0773 [hep-ph].

[32] E. Eichten and F. Feinberg, Phys. Rev. D 23 (1981) 2724; A. Barchielli, N. Brambilla and G. M. Prosperi, Nuovo Cim. A 103 (1990) 59.

[33] N. Brambilla and A. Vairo, arXiv:hep-ph/9904330.

[34] Y. Koma, M. Koma and H. Wittig, arXiv:0711.2322 [hep-lat]. Y. Koma, M. Koma and H. Wittig, Phys. Rev. Lett. 97, 122003 (2006); Y. Koma and M. Koma, arXiv:hep-lat/0609078.; Y. Koma and M. Koma, arXiv:0911.3204 [hep-lat]

[35] N. Brambilla, P. Consoli and G. M. Prosperi, Phys. Rev. D 50 (1994) 5878

[36] M. Laine, O. Philipsen, P. Romatschke and M. Tassler, JHEP 0703 (2007) 054; M. Laine, O. Philipsen and M. Tassler, JHEP 0709 (2007) 066; M. Laine, JHEP 0705 (2007) 028; A. Beraudo, J. P. Blaizot and C. Ratti, Nucl. Phys. A 806, 312 (2008). M. A. Escobedo and J. Soto, arXiv:0804.0691 [hep-ph]; M. A. Escobedo and J. Soto, arXiv:1008.0254 [hep-ph].

[37] N. Brambilla, J. Ghiglieri, A. Vairo and P. Petreczky, Phys. Rev. D 78 (2008) 014017. A. Vairo, PoS C ONFINEMENT8 (2008) 002 N. Brambilla, J. Ghiglieri, P. Petreczky and A. Vairo, Phys. Rev. D 82 (2010) 074019

[38] N. Brambilla, M. A. Escobedo, J. Ghiglieri, J. Soto and A. Vairo, JHEP 1009 (2010) 038; 